\title{
Stem cell gene delivery
}

Dystrophin has been successfully delivered to diseased muscle using intravenous injection of engineered stem cells. The approach represents an alternative to gene delivery via viral/synthetic vector or direct injection into muscle. Scientists at Children's Hospital and Harvard Medical School (Boston, MA) used bone marrow and musclederived stem cells to partially restore dystrophin expression in the $m d x$ mouse model of Duchenne muscular dystrophy (Nature 401, 390-393, 1999). Richard Mulligan, a Howard Hughes investigator and senior author on the study, explains that "the use of stem cells for transplantation appears to result in the disseminated delivery of cells, a critical requirement for the effective treatment of. . .muscular dystrophy, where the afflicted cells are found throughout the body." By irradiating the mice and injecting stem cells engineered to express dystrophin, Mulligan and his colleagues showed that dystrophin-positive muscle cells could successfully engraft into diseased tissue. The approach was successful not only for hematopoietic stem cells, but also for putative muscle tissue stem cells. Although the restorative effects were well below what would be required for clinical efficacy, the findings suggest that adult stem cells from a variety of sources might be manipulated for use in gene therapy protocols.

\section{Deep pocket drugs}

Two teams have reported the development of new anti-HIV compounds that target a highly conserved pocket in the viral coat protein gp41. In one study (Cell 99, 103-115, 1999), scientists at MIT (Cambridge, MA) began by synthesizing the pocket segment of gp41 from D-amino acids, creating a mirror image of the natural pocket. Using this D-peptide as bait, they screened a phage expression library and identified L-peptides that bind specifically to the synthetic pocket. D-peptide versions of these were then synthesized and shown to both bind specifically to the normal gp41 pocket and prevent HIV entry into cells. As D-peptides do not occur naturally and are resistant to proteolytic enzymes, optimized versions of the new pocket-binding peptides might be useful as orally delivered drugs. According to Howard Hughes Investigator Peter Kim, senior author on the study, a nonexclusive license on the technique is currently being offered. A more traditional approach using a rationally designed biased combinatorial library has been employed by researchers at the Howard Hughes Medical Institute, Harvard Medical School (Boston, MA), to identify small molecules that also bind the gp41 pocket and inhibit HIV entry (Nat. Struct. Biol. 6, 953-959, 1999).

Research News Briefs written by Alan Dove, Robert Frederickson, and Andrew Marshall

\section{Potent SOD mimic}

Scientists have identified a fully functional small-molecule mimic of superoxide dismutase (SOD) that is $\sim 60$-fold smaller than the native enzyme (Science 286, 304-306, 1999). When injected intravenously into rats, the mimic prevents inflammatory damage or ischemic injury resulting from superoxide anions. According to Daniela Salvemini and Dennis Riley, senior authors on the paper, a painstaking search of over 100 molecules culminated in the identification of M40403, a molecule with catalytic activity/substrate specificity comparable to the natural enzyme. The authors first chose manganese as the reactive center due to its low toxicity, and then turned to computer modeling and structure-activity relationship analysis to find the surrounding molecular structure. When M40403 was tested in a rat footpad model of inflammation, it prevented both neutrophil infiltration and the production of inflammatory cytokines. Potent antiinflammatory and cytoprotective activities were also demonstrated in a rat model of ischemia-perfusion injury and shock, the mimic significantly prolonging survival time. "We now have a real pharmacological tool to explore the selective role of superoxide in health and disease," says Salvemini. While the current mimics must be injected, orally available versions are also planned by MetaPhore Pharmaceuticals (St Louis, MO).

\section{“Functional” proteomics?}

DNA arrays have opened the door to comparative studies of gene expression on a massively parallel scale. Unfortunately, similar tools for analyzing the proteins on such a global scale are not yet available. In a recent issue of Proc. Natl. Acad. Sci USA (96, 10632-10636, 1999), David Morris and his colleagues have devised a compromise solution to this problem: use DNA arrays to identify those mRNAs undergoing active translation. Using a method called sucrose gradient analysis, populations of cellular mRNAs that are either rapidly or poorly translated are isolated from both quiescent and actively growing cells. DNA probes made from these pools are then applied to commercially available cDNA arrays. About $1 \%$ of the mRNAs were found to be translationally regulated in fibroblasts stimulated with growth factors. "From our standpoint, the real advance of this work is making it easier to look at phenotype", states Morris. He points out that although DNA array technologies still have difficulty detecting very low-level messages, the approach his group has devised is still more sensitive than protein analysis on two-dimensional gels.

\section{Microsequencer put through its paces}

In the first demonstration of its kind, researchers at the Whitehead Institute (Cambridge, MA) have shown that a microdevice etched onto a silica wafer can be used to sequence "real world" DNA samples prepared for the Human Genome Project. The device, consisting of a $12.5 \mathrm{~cm}$ channel and a loading mechanism etched into a silica wafer, achieved $99 \%$ accuracy with samples taken from the production line of the Institute's Center for Genome Research. "We obtain 550 base reads in less than 20 minutes. This performance is quite similar in read length to a Perkin Elmer 3700 [sequencing machine], which requires electrophoresis times. . .exceeding an hour," explains Daniel Ehrlich, senior author on the study (Genome Res. 9, 853-858, 1999). Ehrlich adds that the ability to rapidly sequence comparatively crude samples should prove useful in high-speed forensic analysis: "For DNA forensics, we achieve simple tandem repeat assays in less than 2 minutes [that] are comparable in quality to a 40 minute run on a capillary machine." 